\title{
Subject Index Vol. 33, 1996
}

\section{Acetylcholine 395,463 Actin 13}

Active oxygen species 42 Adhesion molecules $181 \mathrm{c} 1 / 8$-Adrenoceptor 25 B-Adrenoceptors 62, 124 Age 47 Aging 442 Aldosterone

315 Amlodipine 71 Angiogenesis 266, 480 Angioplasty 156, 308 Angiotensin 386

- II 195,480 Antioxidant 42 Aorta 53,235,288

- occlusion 111 Arcade formation 480 Arterial diameter 47

- distensibility 47

- wall 5

ATi receptors 386 Atherogenesis 327 Atherosclerosis 5, 156, 425, 442

- prevention 5 ATP 139

- release 1

BAY K 864432 Bradykinin 340 Buffers 86

$\mathrm{Ca}^{2+}$ channels 258,454

- influx $414{ }^{45} \mathrm{Ca}^{2+}$ uptake 454 Caffeine 489 Calcium 360

- antagonists 5

- channel 25 Canine 53

Capillary resistance 111 Cardiac fibroblasts 315 Cardiovascular disease 139 Carotid artery 288

- - bifurcation 47 Cellmotility 13 Cell-rich plaques 327 Cell-to-cell communication 380

- interactions 327

Cerebral smooth muscle cells 258 cGMP 99, 395

cGMP-dependent protein kinase 99, 386 Chorioallantoic membrane 480

Coculture 327 Collagen expression 315 Collateral resistance 471 Congestive heart failure 62 Contraction 288 Coronary arteries

$78,175,209$

- $\quad$-, canine 62

Cyclic adenosine monophosphate 124

- strain 405

Cytosolic free $\mathrm{Ca}^{2+} 132$

Deoxycorticosterone acetate 235 Desensitization 386 Development 266 Diabetes mellitus 454 Diadenosine polyphosphates 132 Digital imaging microscopy 71

Elastic tissue 425

Elastin 195

Electrophysiology 380

Embryo, chick 480

Endothelial cell(s) 53, 139, 146, 225, 327 ,

360, 405 Endothelin 71, 139,425 Endothelium 13, 62, 78, 164, 347, 425, 463 Endothelium-dependent relaxation 414

Endothelium-derivedhyperpolarization

380 - hyperpolarizing factor 119,225 Endotoxin 249 En face immunohistochemical technique

425 Estrogen 124 Exercise training 266 Extracellular matrix 433

Femoral artery bifurcation 47

Fibrosis 315

Flow 119

-, pulsatile 360

-, steady 360

Free radicals 463

Fura-2 489 
G protein 25 Gap junctions 395 Gene expression 195 Glutathione peroxidase 42 Glycosaminoglycans 433 Growth factors 266 Guanylate cyclase 299 Guinea pig heart 1

[3H]-(+)-PN200-1 10 binding 454

Heart disease 209

Hemolysate 258

Heparan sulfate 433

Heptanol 395

Heterologous desensitization 489

Hirulog 308

Hormones 266

Hypercapnia 347

Hyperlipidemia 442

Hyperplasia 266

Hypertension $164,175,195,235,425$

Hypertrophy 266

Immature collaterals 471

Immunohistochemistry 146,425

Increased flow 1

Inhibition 71

In situ hybridization 195

Intimal cushions 209

- hyperplasia 146,405

- proliferation 42 Intracellular calcium 386 Ions 86

Isoforms, myosin 442 Isolated kidney 119 Isometric force 489 Isoprenaline 124 Isoproterenol 62

Kinins 380

Leucocytes 425

Ligands 86

Lipid peroxidation 463

Lipid-rich plaques 327

Lipopolysaccharides 370

Lipoproteins 327

Low density lipoprotein 181

Macrophages 5, 370 Malondialdehyde 463 Mature collaterals 471 Mechanical deformation 405

- factors 266

- properties 175 Membrane depolarization 454

- potential 414 Mesenteric arteries 235

- bed 370

- resistance arteries 195 Methylene blue 340 Mibefradil 71

Microfilaments 13

Microtubules 13

Mineralocorticoid receptor mRNA 315

Mitogen-activated protein kinase 288

Myogenic response 32

Natriuretic peptide 78

Neointima 146

Nifedipine 32

Nitric oxide 78, 119, 181, 225, 249, 340,

$370,386,395,471$ Nitroglycerin tolerance $78 \mathrm{~N}^{\omega}$-Nitro-L-arginine 340 - methyl ester 119,471 Nonneuronal cell memory 489 Nuclear factor kappa B 181

Octanol 395

Oscillation 360

Osteopontin 195

Ouabain-sodium pump activity 164

Passive recoil 111 Pertussis toxin $25 \mathrm{pH} 347$ 
Phenylephrine 32,489 Phosphoprotein 99 Phosphorylation 99 Piglet 249 Pigs 175

PKC translocation 71 Platelet adhesion 156

Platelet-derived growth factor 53

Polyploidy 195

Porcine 13

Potassium 32

Pressurized mesenteric arteries 124

Proliferation 146,405

Prostaglandins 480

Pulmonary artery 249

Rabbit 146, 156

- femoral artery 489

- model 308 Rat 425

- aorta 164

- mesenteric resistance artery 386

- tail arteries 463 -, Wistar 442 Reactive hyperemia 111 Recoverage 146 Remodelling 175 Restenosis 308

Salt 235

Serotonin (5-HT) receptor 288 Small arteries 32, 235 Smooth muscle 99,209, 347

- - cell(s) 5, 53, 146, 327, 405, 442

Solutions 86

Substance P 139 Substrate specificity 99 Sucrose 395

Superoxide anion 181 Sympathectomy, long-term 139

Tetraethylammonium 119 Thiobarbituric-acid-reactive substance 42 Thrombin 308 Tyrosine kinase(s) 225, 288

- phosphatases 225

U46619 249 Ultrasound 47 Ultrastructure 209 Ultraviolet light 299 Umbilical artery 156

Vascular contraction 454

- remodeling 195,235

- resistance 1

- smooth muscle 25, 32, 124, 195, 288, 299, 380, 395, 489

- - - cells 132,433

Vasoconstriction 119

Vasodilation 111

Vasodilator 71

Vasomotor tone 370

Vasospasm 258

Vein graft 405

Vinculin 13

Voltage-operated $\mathrm{Ca}^{2+}$ channel 32

Wall tracking 47 Wound 13 
Subject Index Vol. 33, 1996 